

Short Communication

\title{
DNA sequence variants in the carbonyl reductase 1 (cbr1) gene in seven breeds of Canis lupus familiaris
}

\author{
Q. Cheng, C. Sanborn, D. Ferguson and J.G. Blanco \\ Department of Pharmaceutical Sciences, \\ The State University of New York at Buffalo, Buffalo, NY, USA
}

Corresponding author: J.G. Blanco

E-mail: jgblanco@buffalo.edu

Genet. Mol. Res. 11 (2): 1109-1116 (2012)

Received September 21, 2011

Accepted December 22, 2011

Published April 27, 2012

DOI http://dx.doi.org/10.4238/2012.April.27.10

\begin{abstract}
The anticancer anthracyclines doxorubicin and daunorubicin are used to treat a variety of cancers in dogs. The therapeutic utility of anthracyclines is limited by cardiotoxicity in some cases. Synthesis of anthracycline alcohol metabolites by carbonyl reductase 1 (CBR1) is crucial for the pathogenesis of cardiotoxicity. We hypothesize that genetic polymorphisms in canine $c b r l$ contribute to the variable pharmacodynamics of anthracyclines in dogs. DNA sequence variants in canine $c b r l$ were investigated in DNA samples from dogs of seven breeds. Thirteen SNPs were detected in canine cbrl. A 10-bp deletion in the 5'-untranslated region (5'UTR) was found in specimens from the Labrador Retriever, Beagle, Siberian Husky, and Boxer breeds. The 5'-UTR also included a polymorphic "hot spot" region immediately downstream of the 10-bp
\end{abstract}


deletion. DNA sequence variants in the "hot spot region" ranged from 1 to $21 \mathrm{bp}$ in length. Bioinformatics searches identified a cluster of three to six potential binding sites for the transcription factor Sp1 in the DNA segment containing both the "hot spot" region and the 10-bp deletion. This information provides a foundation to allow us to investigate whether DNA sequence variants in the 5'-UTR of canine $c b r l$ impact the pharmacodynamics of anticancer anthracyclines in dogs.

Key words: Anthracycline-related cardiotoxicity; Anthracyclines; Domestic dog (Canis lupus familiaris); Carbonyl reductase 1 (cbrl); Cancer; Doxorubicin

\section{INTRODUCTION}

The anticancer anthracyclines doxorubicin and daunorubicin are used to treat a variety of cancers in dogs including lymphomas, hemangiosarcoma, osteosarcoma, mammary tumors, and squamous cell carcinomas (Simon et al., 2006). The therapeutic utility of anthracyclines is limited by the development of cardiotoxicity in some animals. The average incidence of anthracycline-related cardiotoxicity in dogs is $\approx 18 \%$, and cardiotoxicity has been documented in up to $69 \%$ of animals (Gillings et al., 2009). Anthracycline-related cardiotoxicity may induce sudden death by arrhythmias, or it may culminate in congestive heart failure (Herman et al., 1983; Herman and Ferrans, 1998; Morrison, 2002; Astra et al., 2003). To the best of our knowledge, body weight is the only documented potential risk factor for anthracycline-related cardiotoxicity in dogs (Gillings et al., 2009). Additional risk factors for anthracycline-related cardiotoxicity in dogs remain to be defined.

The pathogenesis of anthracycline-related cardiotoxicity is mediated by a combination of oxidative stress and metabolic perturbations induced by $\mathrm{C}-13$ anthracycline alcohol metabolites (e.g., doxorubicinol and daunorubicinol) (Minotti et al., 2004). Carbonyl reductase 1 (CBR1) catalyzes the synthesis of cardiotoxic C-13 alcohol metabolites. The role of CBR1 activity during the pathogenesis of anthracycline-related cardiotoxicity has been firmly established through biochemical and genetic studies in mice, humans and rabbits (Forrest et al., 2000; Mordente et al., 2001; Olson et al., 2003). Interestingly, single nucleotide polymorphisms (SNPs) in the human CBRs impact the pharmacodynamics of anthracyclines and the risk of anthracycline-related cardiotoxicity (Lal et al., 2008; Blanco et al., 2008, 2011). We hypothesize that SNPs in canine $c b r 1$ may contribute to the variable pharmacodynamics of anthracyclines in dogs. Here, we documented DNA sequence variants in canine cbrl by analyzing DNA samples from dogs of 7 breeds.

\section{DNA samples}

DNA samples from unrelated Labrador Retrievers $(\mathrm{N}=14)$, German Shepherds $(\mathrm{N}=15)$, Beagles $(\mathrm{N}=13)$, Boxers $(\mathrm{N}=14)$, Poodles $(\mathrm{N}=12)$, Siberian Huskies $(\mathrm{N}=$ $15)$, and Golden Retrievers $(\mathrm{N}=14)$ were acquired from the CHIC repository (Orthopedic 
Foundation for Animals, OFFA). DNA was isolated from peripheral blood using standard methodology.

\section{Detection of $c b r 1$ polymorphisms}

Canine $\operatorname{cbr} 1$ exons 1, 2, 3 and corresponding flanking sequences (Canis lupus familiaris, chromosome 31, GenBank reference sequence NC_006613.2) were amplified by polymerase chain reaction (Appendix 1). Amplification products were sequenced with forward and reverse primers in an Applied Biosystems 3130xl Genetic Analyzer. SNP analysis was performed with a web-based tool (http://lpgws.nci.nih.gov/perl/snp/snp_cgi.pl). The presence of SNPs, nucleotide insertions and nucleotide deletions was verified by visual inspection of the electropherograms.

\section{RESULTS AND DISCUSSION}

The 3 exons of canine $c b r 1$ and up to 359 bp from the 5'-untranslated region (5'UTR) were amplified to analyze the presence of DNA sequence variants in the coding and potential promoter regions, respectively. Thirteen SNPs were detected in canine $c b r 1$, including 2 synonymous SNPs, 7 SNPs in the 5'-UTR and 4 intronic SNPs (Tables 1 and 2). A 10-bp deletion in the 5'-UTR was present in specimens from the Labrador Retriever, Beagle, Siberian Husky, and Boxer breeds. The 5'-UTR included a polymorphic "hot spot" region immediately downstream of the 10-bp deletion. The "hot spot" included sequence variants ranging from 1 to $21 \mathrm{bp}$ in length. None of the 61 DNA samples (including 11 samples from Boxer dogs) showed the corresponding 10-bp fragment (5'-CACGAGACCC-3') reported in the GenBank reference sequence from a Boxer dog (Table 1). These findings highlight the polymorphic nature of the dog's genome.

Bioinformatics searches focused on the proximal 5'-UTR of canine cbrl (400 bp screened) pinpointed a cluster of 3 to 6 conserved motifs for the transcription factor Sp1 (5'-GCCACGCC-3') (McDowell et al., 2005). The number of Sp1 motifs, 10-bp deletion status (yes/no), and corresponding DNA sequences for the "hot spot" region are listed in Table 3. Of note, the Sp1 cluster is located in the DNA segment containing both the "hot spot" region and the polymorphic 10-bp deletion (length: 44 to $64 \mathrm{bp}$ ). In this segment, the GenBank reference sequence has a total of 4 Sp1 motifs (Table 3). Interestingly, one Sp1 motif is abrogated by the 10-bp deletion. In contrast, the presence of "hot spot" variants 3 , 5,7 , and 9 resulted in an additional Sp1 motif. Similarly, 2 additional Sp1 motifs were created when the relatively long "hot spot" variant 8 (length: $21 \mathrm{bp}$ ) was present (Tables 1 and $3)$. Our previous studies have shown that the aryl hydrocarbon receptor pathway regulates the expression of human CBRI through conserved xenobiotic response elements (XREs) in the gene promoter region (Lakhman et al., 2007). Here, we pinpointed a conserved $X R E$ (5'-CACGCCA-3') in the vicinity of the Sp1 cluster in the 5'-UTR of canine cbrl (306 bp upstream of the translation initiation codon, $\left.\mathrm{A}_{+1} \mathrm{TG}\right)$. Future studies are warranted to investigate whether the $X R E$, in concert with the variable number of Sp1 motifs, impacts the transcription of canine $c b r l$ and, consequently, the pharmacodynamics of anticancer anthracyclines in dogs (Wang et al., 1998). 
Table 1. DNA variants in canine cbrl (5'-UTR and exon 1) - Canis lupus familiaris chromosome 31, whole genome shotgun sequence (NCBI reference sequence: NC_006613.2)*.

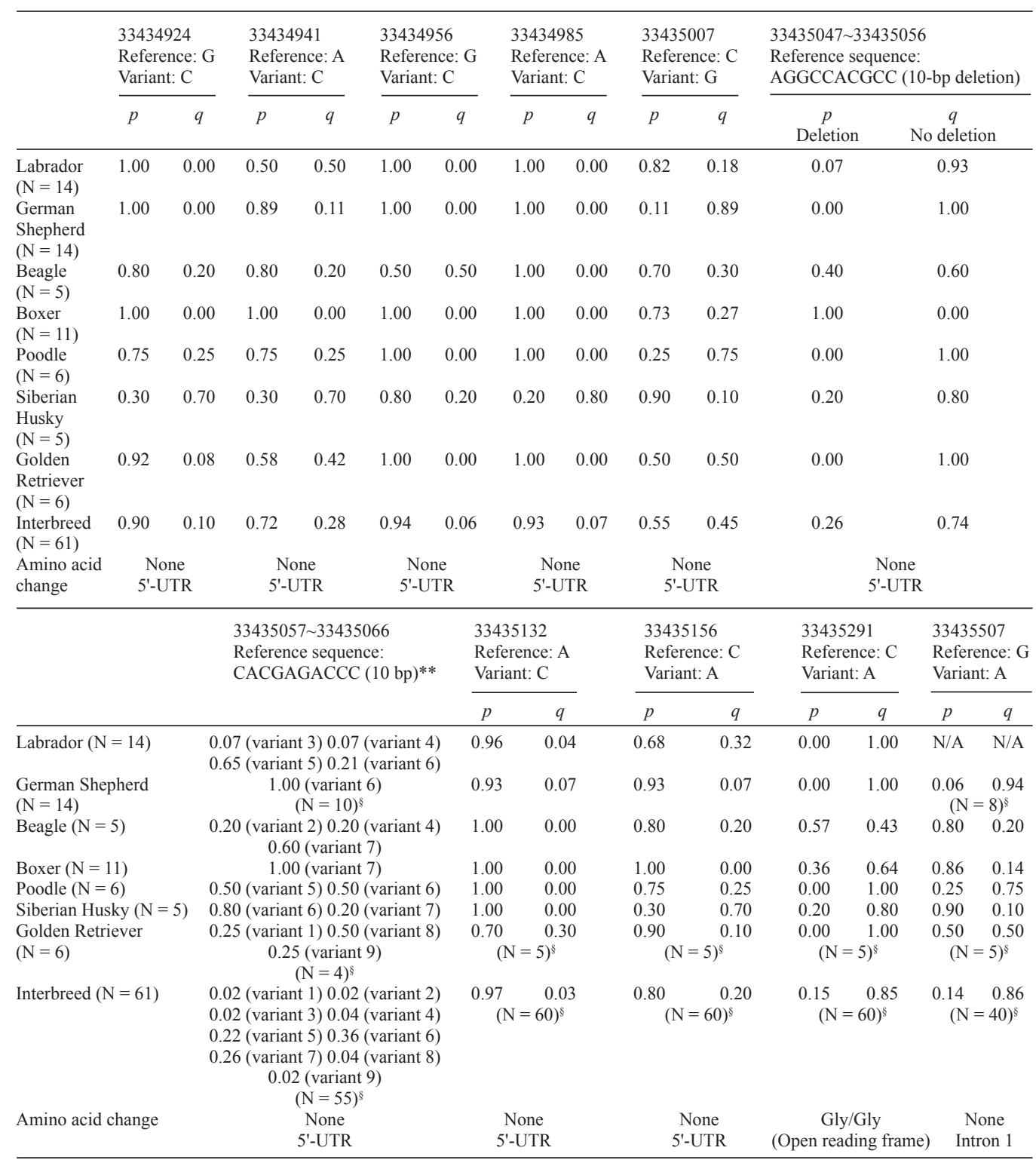

*GenBank reference sequence from a Boxer dog (http://www.ncbi.nlm.nih.gov/genome/guide/dog/). The translation initiation codon $\left(\mathrm{A}_{+1} \mathrm{TG}\right)$ is located at positions: $33435163-33435165$. **Variant 1: 5'-C-3' (1 bp); variant 2: 5'-CAGGCCACG-3' (9 bp); variant 3: 5'-AGGCCACGCC-3' (10 bp ); variant 4: 5'-CAGACCACGC-3' (10 bp); variant 5: 5'-CAGGCCACGCC-3' (11 bp); variant 6: 5'-CAGACCACGCC-3' (11 bp); variant 7: 5'-CTGGCCACGCC-3' (11 bp); variant 8: 5'-CAGGCCACGCCTAGCCACGCC-3' (21 bp); variant 9: 5'-CAGACCACGCCTAGCCACGCC-3' (21 bp). N/A = not analyzed. \$In some cases, the number of samples analyzed was different from the totals shown in the first column. $p$ and $q=$ allele frequencies. 
DNA sequence variants in the $c b r l$ gene in C. l. familiaris
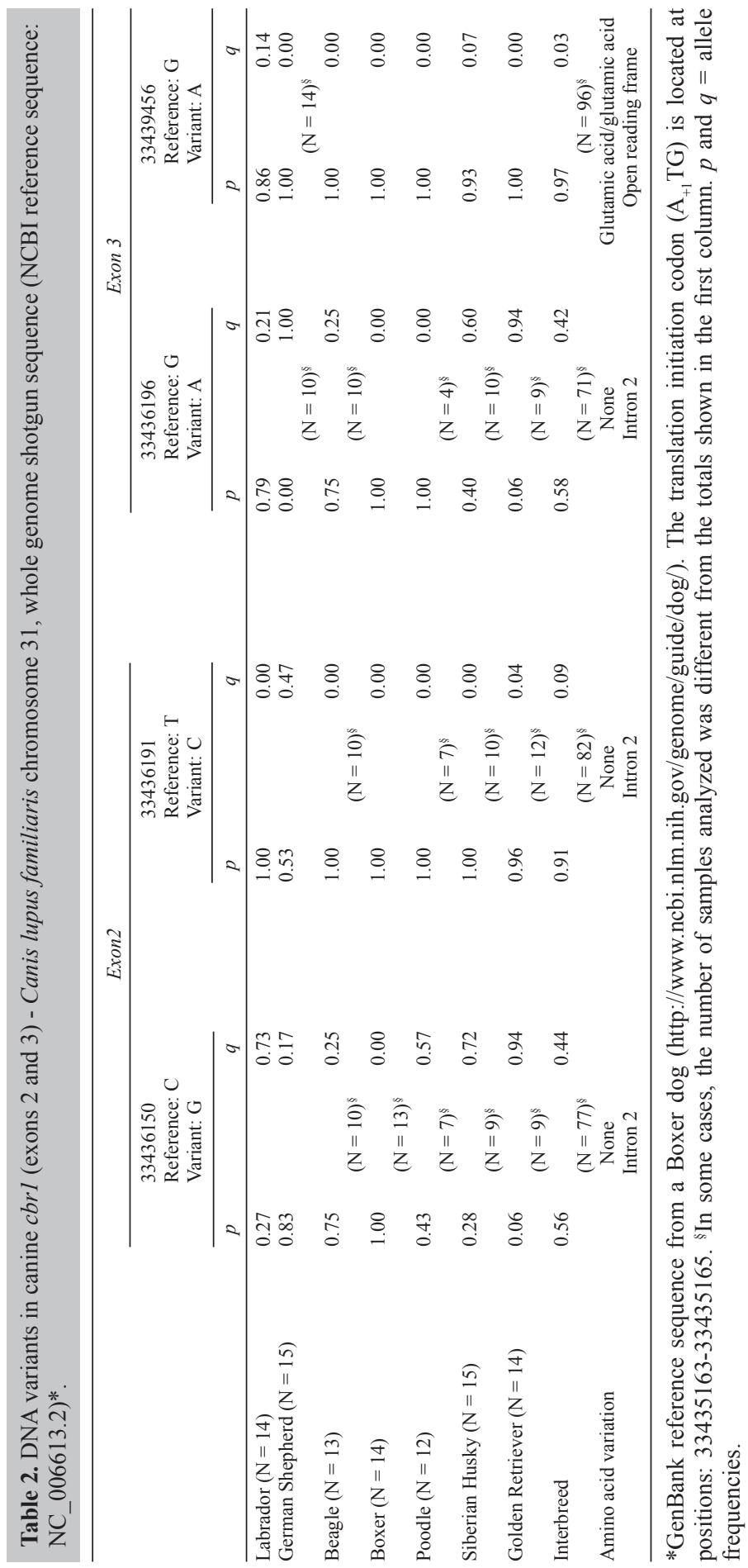
Q. Cheng et al.

1114

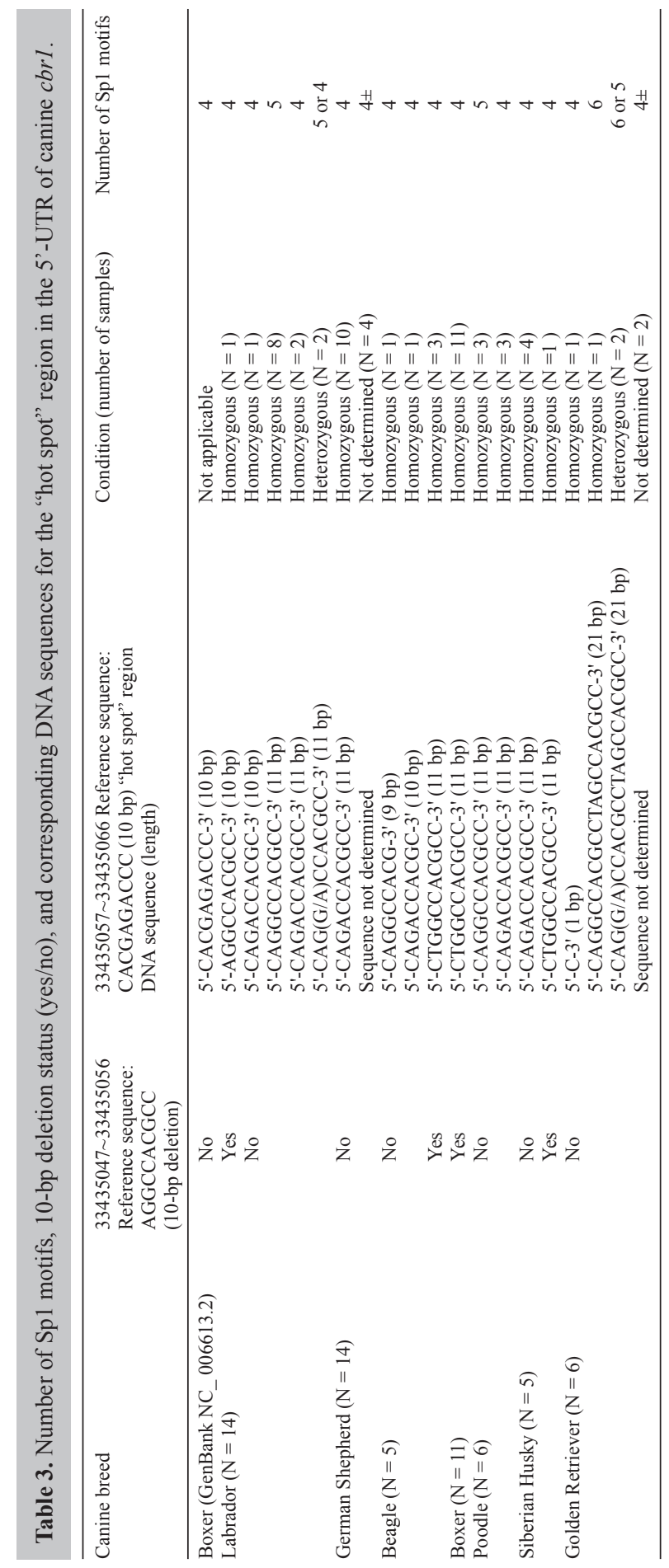




\section{ACKNOWLEDGMENTS}

Research supported by the National Institutes of Health/National Institute of General Medical Sciences (Grant \#GM73646). We thank Eddie Dziuk (OFFA) and Dr. Christine Haakenson (Canine Health Foundation) for providing precious dog DNA samples.

\section{REFERENCES}

Astra LI, Hammond R, Tarakji K and Stephenson LW (2003). Doxorubicin-induced canine CHF: advantages and disadvantages. J. Card. Surg. 18: 301-306.

Blanco JG, Leisenring WM, Gonzalez-Covarrubias VM, Kawashima TI, et al. (2008). Genetic polymorphisms in the carbonyl reductase 3 gene $\mathrm{CBR} 3$ and the $\mathrm{NAD}(\mathrm{P}) \mathrm{H}$ :quinone oxidoreductase 1 gene $\mathrm{NQO} 1$ in patients who developed anthracycline-related congestive heart failure after childhood cancer. Cancer 112: 2789-2795.

Blanco JG, Sun CL, Landier W, Chen L, et al. (2011). Anthracycline-related cardiomyopathy after childhood cancer: role of polymorphisms in carbonyl reductase genes - A report from the Children's Oncology Group. J. Clin. Oncol. (in press) DOI [10.1200/JCO.2011.34.8987].

Forrest GL, Gonzalez B, Tseng W, Li X, et al. (2000). Human carbonyl reductase overexpression in the heart advances the development of doxorubicin-induced cardiotoxicity in transgenic mice. Cancer Res. 60: 5158-5164.

Gillings S, Johnson J, Fulmer A and Hauck M (2009). Effect of a 1-hour IV infusion of doxorubicin on the development of cardiotoxicity in dogs as evaluated by electrocardiography and echocardiography. Vet. Ther. 10: 46-58.

Herman EH and Ferrans VJ (1998). Preclinical animal models of cardiac protection from anthracycline-induced cardiotoxicity. Semin. Oncol. 25: 15-21.

Herman EH, Rahman A, Ferrans VJ, Vick JA, et al. (1983). Prevention of chronic doxorubicin cardiotoxicity in beagles by liposomal encapsulation. Cancer Res. 43: 5427-5432.

Lakhman SS, Chen X, Gonzalez-Covarrubias V, Schuetz EG, et al. (2007). Functional characterization of the promoter of human carbonyl reductase 1 (CBR1). Role of XRE elements in mediating the induction of CBR1 by ligands of the aryl hydrocarbon receptor. Mol. Pharmacol. 72: 734-743.

Lal S, Sandanaraj E, Wong ZW, Ang PC, et al. (2008). CBR1 and CBR3 pharmacogenetics and their influence on doxorubicin disposition in Asian breast cancer patients. Cancer Sci. 99: 2045-2054.

McDowell TL, Symons JA and Duff GW (2005). Human interleukin-1 alpha gene expression is regulated by Sp1 and a transcriptional repressor. Cytokine 30: 141-153.

Minotti G, Menna P, Salvatorelli E, Cairo G, et al. (2004). Anthracyclines: molecular advances and pharmacologic developments in antitumor activity and cardiotoxicity. Pharmacol. Rev. 56: 185-229.

Mordente A, Meucci E, Martorana GE, Giardina B, et al. (2001). Human heart cytosolic reductases and anthracycline cardiotoxicity. IUBMB Life 52: 83-88.

Morrison WB (2002). Cancer in Dogs and Cats: Medical and Surgical Management. Teton New Media, Jackson Hole.

Olson LE, Bedja D, Alvey SJ, Cardounel AJ, et al. (2003). Protection from doxorubicin-induced cardiac toxicity in mice with a null allele of carbonyl reductase 1. Cancer Res. 63: 6602-6606.

Simon D, Nolte I, Eberle N, Abbrederis N, et al. (2006). Treatment of dogs with lymphoma using a 12-week, maintenancefree combination chemotherapy protocol. J. Vet. Intern. Med. 20: 948-954.

Wang F, Hoivik D, Pollenz R and Safe S (1998). Functional and physical interactions between the estrogen receptor Sp1 and nuclear aryl hydrocarbon receptor complexes. Nucleic Acids Res. 26: 3044-305. 
Q. Cheng et al.

1116

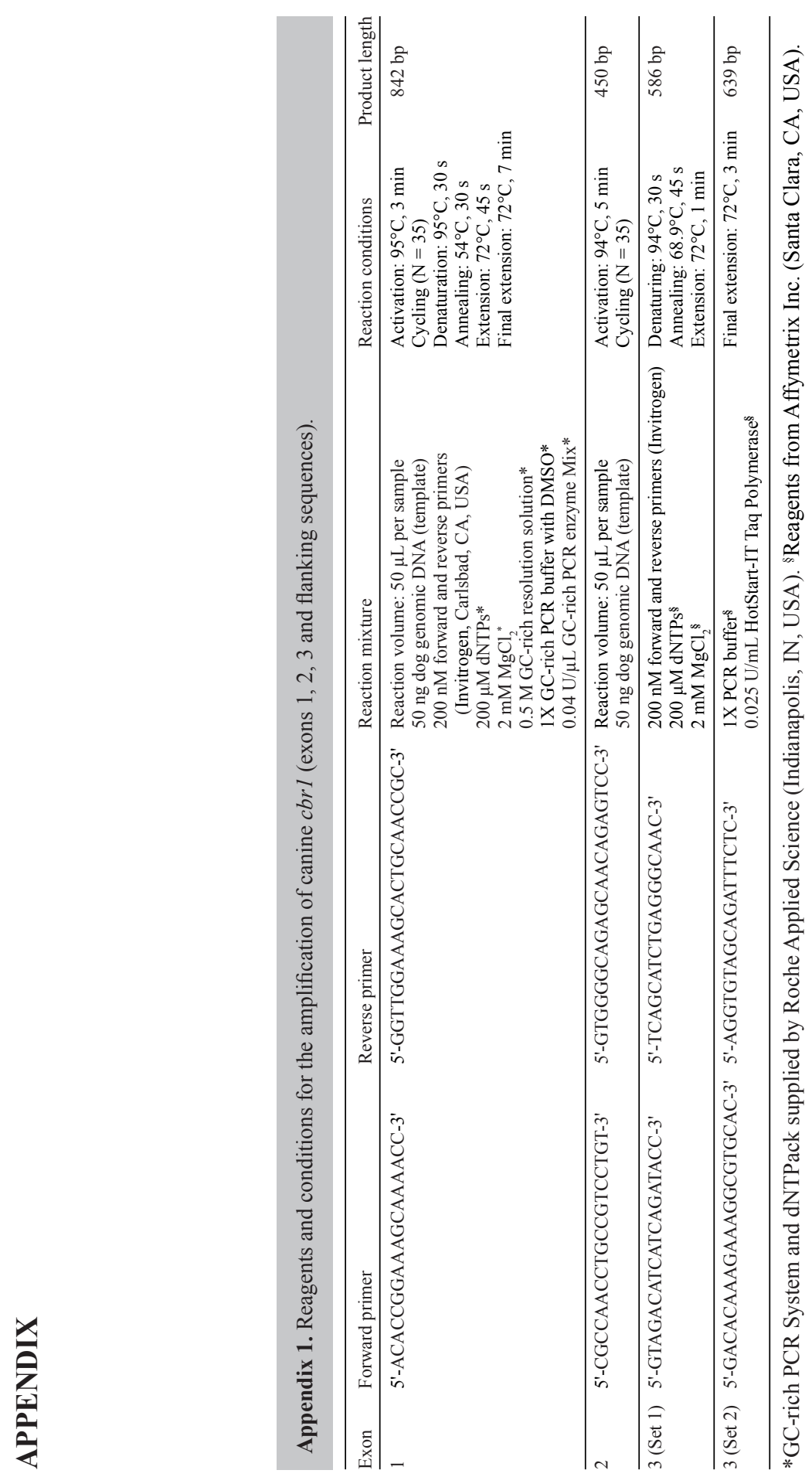

Genetics and Molecular Research 11 (2): 1109-1116 (2012) 